\title{
¿Deben incluirse los nombres de líquidos en Terminología Anatómica?
}

\author{
${ }^{\circledR}$ Rafael Coello Cuntó ${ }^{1}{ }^{\oplus}$ Rafael Coello Salguero $2,{ }^{\bullet}$ Gary Muñoz Sánchez ${ }^{3}{ }^{\oplus}$ Rita Vega Cobos ${ }^{4}{ }^{\oplus}$ José Véliz Ortega ${ }^{5}$, \\ José Ignacio Macías Alcivar ${ }^{6},{ }^{\oplus}$ Marcos Jiménez Asang ${ }^{1},{ }^{\circ}$ Yodrany Jiménez Asang ${ }^{1}$.
}

\section{RESUMEN}

T as denominaciones anatómicas humanas son el punto de partida para ordenar todo idioma científico biomédico y la _comunicación científica está subordinada a Terminología Anatómica (TA). Para la incorporación de un término anatómico se utilizan resoluciones provenientes de la FIPAT y principios universales como el respeto a la identidad semántica de la estructura y sus componentes; sin embargo, en TA existen términos de líquidos biológicos y la ausencia de otros; los líquidos son resultado de procesos metabólicos y participan en fenómenos fisiológicos. En la publicación de la FIPAT 2da edición, Terminología Anatómica, 2011 se incluyen denominaciones de líquidos sin precisar el criterio de selección. Este documento pretende establecer una conciliación de dicha inequidad incorporando términos de líquidos biológicos básicos; se propone la necesidad de incorporar en un anexo dichos términos en atención a su presencia en espacios reales o virtuales sin aumentar el número de términos enlistados en el texto.

PALABRAS CLAVE: Terminología Anatómica (TA), Programa Federativo Internacional de Terminología Anatómica (FIPAT), Comité Federativo de Terminología Anatómica (FCAT), líquidos biológicos.

\section{Are the fluid terms should be included in Anatomical Terminology?}

\section{ABSTRACT:}

Чuman anatomical denominations are the starting point for ordering all biomedical scientific language and scientific - communication is subordinated to Anatomical Terminology (AT). For the incorporation of an anatomical term, resolutions from FIPAT and universal principles such as respect for the semantic identity of the structure and its components are used; however, in AT there are terms of biological liquids and the absence of others; liquids are the result of metabolic processes and participate in physiological phenomena. In the FIPAT 2nd edition publication, Anatomical Terminology, 2011, liquid terms are included without specifying the selection criteria. This document intends to establish a reconciliation of such inequity by incorporating basic biological liquid terms; it is proposed the need to incorporate in an annex such terms in view of their presence in real or virtual spaces without increasing the number of terms listed in the text.

KEY WORDS: Anatomical Terminology (AT), International Federative Program for Anatomical Terminology (FIPAT), Federative Committee for Anatomical Terminology (FCAT), biological liquids.

\section{INTRODUCCIÓN}

$\mathrm{D}$ esde Basilea (1895) se describe la estructura humana en la Nómina Anatómica (BNA); luego revisada en Birminghan en 1933, en 1936 se aprueba la Nómina Anatómica de Jena (JNA); en 1955 se adopta la nomenclatura latina y se publica en Paris la 1ra edición de la Nómina Anatómica (PNA) basada en la publicación de Basilea; es revisada en 1961(2da edición)1966 (3ra edición)1977 (4ta edición)1983 (5ta edición) y1989 (6ta edición); como consecuencia de la organización de la FCAT en1998 se publica la TAI (Terminología Anatómica Internacional) (1) que en Septiembre del 2001 es traducida al español (2). Cada edición ha reiterado la preocupación de describir de manera científica, sencilla, exacta y descriptiva la morfología humana. Revisando el texto de terminologia anatomica (TA) de la FCAT, 2001 y de la FIPAT, TA
2011 se aprecia que desde los capítulos de generalidades la intención es respetar los principios de posición anatómica, planigrafía, incluyéndose términos de dirección y denominaciones funcionales. Los textos de Morfología se refieren a un ser humano inmóvil, inerte $\mathrm{y}$ en condiciones de bionormalidad y mencionan todos los líquidos sin excepción en sus descripciones; sin embargo, en los textos de TA se aceptan ciertos términos de líquidos y otros están excluidos sin establecer las razones.

Por otro lado, el número de términos ha disminuido considerablemente y cada vez se están optimizando de acuerdo a las recomendaciones que la FIPAT, organismo acreditado por la comunidad científica para aceptar recomendaciones e incorporaciones

\footnotetext{
1. Universidad de Guayaquil, Ecuador.

2. Hospital de Especialidades Teodoro Maldonado Carbo (IESS), Guayaquil -.Ecuador.

Hospital Sagrado Corazón de Jesús, Quevedo - Ecuador

Aeropuerto Ecológico de Galápagos.

Hospital de Especialidades Abel Gilbert Pontón, Guayaquil -.Ecuador.

Gobierno Autónomo Descentralizado Municipio de Playas- Ecuador

Citar como : Coello Cuntó R, Coello Salguero R, Muñoz Sánchez G, Vega Cobos R, Velíz Ortega J, Macías Alcivar JI, Jiménez Asang M, Jiménez Asang Y. ¿Deben incluirse los nombres de líquidos en Terminología Anatómia?. Rev Peruana de Morfologia. 2020; 1(1): 7-9

Recibido: 19/05/2020; Aceptado: 12/08/2020

Autor corresponsal: Rafael Coello Cuntó Phd., Mg. Esp; rafael.coelloc@ug.edu.ec
} 
por medio del análisis científico de sus organismos regionales como el Simposio Iberolatinoamericano de Terminología Anatómica (SILAT) (3). El propósito de este documento es analizar la presencia de términos de líquidos en TA, su identidad lexicográfica en latín y considerar la posibilidad de revisar otros términos con las mismas características físicas, considerando su papel fundamental en el metabolismo humano; el propósito es optimizar Terminología Anatómica buscando una frontera científica y conceptual que defina lo anatómico $\mathrm{y}$ lo funcional $\mathrm{y}$ no cometer injustas exclusiones $\mathrm{o}$ confusas inclusiones.

\section{MATERIAL}

Para el análisis se considerarán solo los términos de líquidos incluidos en los textos TA del 2001 y del 2011; aceptándose como tales aquellos que cumplen características físicas de fluidez, se adjuntará su declinación latina, caso y género correspondientes. El primer listado (Tabla 1) presenta 7 términos que son referidos en los textos con sus correspondientes códigos alfanuméricos y el siguiente listado (Tabla 2) contiene 10 términos que se analizarán con las mismas consideraciones y se encuentran sin codificar.

Tabla 1. Términos de líquidos biológicos declarados en TA

\begin{tabular}{|c|c|c|c|c|c|c|c|}
\hline \multicolumn{2}{|r|}{ Término en latín } & Inglés & \multirow{2}{*}{$\begin{array}{r}\text { Español } \\
\text { Líquido sinovial }\end{array}$} & \multicolumn{2}{|c|}{ Género } & \multirow{2}{*}{$\frac{\text { Caso }}{\text { Nominativo }}$} & \multirow{2}{*}{$\frac{\text { Declinación }}{\text { 1ra }}$} \\
\hline A03.0.00.031 & Synovia & Synovial fluid & & $\mathrm{S}$ & $\mathrm{F}$ & & \\
\hline A12.0.00.009 & Haema, sanguisinis (m) & Blood & Sangre & S & $\mathrm{F}$ & Nominativo & 1ra \\
\hline A12.0.00.043 & Lympha & Lymph & Linfa & S & $\mathrm{F}$ & Nominativo & $1 \mathrm{ra}$ \\
\hline A14.1.01.203 & Liquor cerebroespinalis & $\begin{array}{l}\text { Cerebrospinal } \\
\text { fluid }\end{array}$ & Líquido cerebroespinal & $\mathrm{S}$ & M & $\begin{array}{l}\text { Nominativo } \\
\text { Genitivo }\end{array}$ & $\begin{array}{l}3 \mathrm{ra} \\
3 \mathrm{ra}\end{array}$ \\
\hline A15.2.06.002 & Humor aquosus & $\begin{array}{l}\text { Aqueous } \\
\text { humor }\end{array}$ & $\begin{array}{l}\text { Humor } \\
\text { Acuoso }\end{array}$ & $\begin{array}{c}\mathrm{S} \\
\mathrm{Ad}\end{array}$ & M & Nominativo & $\begin{array}{l}3 \mathrm{ra} \\
2 \mathrm{da}\end{array}$ \\
\hline A15.3.03.056 & Perilynpha & Perilymph & Perilinfa & S & $\mathrm{F}$ & Nominativo & $1 \mathrm{ra}$ \\
\hline A15.3.03.061 & Endolynpha & Endolymph & Endolinfa & S & $\mathrm{F}$ & Nominativo & 1ra \\
\hline
\end{tabular}

Tabla 2. Términos de líquidos biológicos no declarados en TA

\begin{tabular}{|c|c|c|c|c|c|}
\hline Español & Inglés & Latín & Caso & Género & Declinación \\
\hline Líquido pleural & Pleural fluid & $\begin{array}{c}\text { Liquor } \\
\text { pleuralis }\end{array}$ & $\begin{array}{c}\text { Nominativo } \\
\text { Genitivo }\end{array}$ & $\begin{array}{l}\mathrm{M} \\
\mathrm{M}\end{array}$ & $\begin{array}{l}3 \mathrm{er} \\
\text { 3er }\end{array}$ \\
\hline Líquido pericardico & Pericardical fluid & $\begin{array}{c}\text { Liquor } \\
\text { pericardiae }\end{array}$ & $\begin{array}{c}\text { Nominativo } \\
\text { Genitivo }\end{array}$ & $\begin{array}{l}\mathrm{M} \\
\mathrm{M}\end{array}$ & $\begin{array}{l}3 \mathrm{er} \\
3 \mathrm{er}\end{array}$ \\
\hline Líquido peritoneal & Peritoneal fluid & $\begin{array}{c}\text { Liquor } \\
\text { peritonealis }\end{array}$ & $\begin{array}{c}\text { Nominativo } \\
\text { Genitivo }\end{array}$ & $\begin{array}{l}\mathrm{M} \\
\mathrm{M}\end{array}$ & $\begin{array}{l}3 \mathrm{er} \\
3 \mathrm{er}\end{array}$ \\
\hline Líquido vaginal & Vaginal fluid & $\begin{array}{c}\text { Liquor } \\
\text { vaginalis }\end{array}$ & $\begin{array}{c}\text { Nominativo } \\
\text { Genitivo }\end{array}$ & $\begin{array}{l}\mathrm{M} \\
\mathrm{F}\end{array}$ & $\begin{array}{l}3 \mathrm{er} \\
3 \mathrm{er}\end{array}$ \\
\hline Bilis & Bile & Bilis & Nominativo & $\mathrm{F}$ & $3 \mathrm{ra}$ \\
\hline Lágrima & Tear & Lacrima & Nominativo & $\mathrm{F}$ & $1 \mathrm{ra}$ \\
\hline Sémen & Semen & Semen & Nominativo & M & $3 \mathrm{ra}$ \\
\hline Orina & Urine & Urina & Nominativo & F & $1 \mathrm{ra}$ \\
\hline Saliva & Saliva & Saliva & Nominativo & $\mathrm{F}$ & 1ra \\
\hline Sudor & Sweat & Sudor & Nominativo & M & $3 \mathrm{ra}$ \\
\hline
\end{tabular}

\section{DISCUSIÓN}

En el cuerpo humano existen áreas, forámenes, meatos, cavidades, surcos, canales, compartimientos, todos espacios visibles y fáciles de identificar; todos ocupados por vasos, nervios, vísceras, tejido adiposo y masas musculares; a su vez cada espacio y sus contenidos tienen una denominación propia y tienen códigos en TA; también existen estructuras saculares pequeñas y otros amplios, con grandes espacios virtuales cubiertos por hojas membranosas que elaboran productos para mantener la bioestabilidad humana. Los líquidos ocupan tanto espacios reales como virtuales; además se conoce que en ciencias biomédicas todos los líquidos biológicos son producto de la actividad hemodinámica, secreción o metabolismo endógeno y/o de filtrados; la sangre es probablemente el inicio de todos los procesos y consta en el texto (4)

Rev. Peruana de Morfologia. 2020; 1(1):7-9
A pesar que a través del tiempo el número de términos ha aumentado de 4311 de la BNA hasta 7635 de TA, la tendencia actual en este campo ha sido el disminuir (suprimiendo los sinónimos) u optimizar el número de términos sin desmedro de lesionar la descripción humana especialmente para al lector que no conoce la anatomía humana; para las ciencias biomédicas, cada término ofrece intrínsecamente información y conocimiento científico de la morfología humana. Los líquidos biológicos forman parte de la estructura y constituyen un componente fundamental de su dinamia. En los textos de TA (5)(6) y Terminología Histológica (TH ) (7), se señalan algunos términos de líquidos biológicos (líquido sinovial, sangre , linfa, liquido cerebroespinal, humor acuoso, perilinfa y endolinfa) que constan en distintas secciones (sinovia en sistema articular) (linfa y sangre en sistema cardiovascular) (líquido cerebroespinal en sistema nervioso) (humor acuoso, perilinfa y endolinfa en órganos de los sentidos) 
pero corresponden y relacionan en dichos sistemas con estructuras estableciendo una clara idea morfo-funcional al indicarlos como términos dependientes de sus hiperónimos (8) entendidos como "parte de" ("liquido sinovial" de la cápsula articular, "sangre" y "linfa" en relación a sus términos de jerarquía "vaso sanguíneo" y "vaso linfático", observándose una clara relación de contenido y continente entre "líquido cerebroespinal" y "espacio subaracnoideo" así como entre "perilinfa" y "espacio perilinfático", "endolinfa" y "espacio endolinfático"; sus definiciones en latín, inglés y español revelan el estado líquido independientemente de su volumen, localización, composición y concentración bioquímica, por el hecho de ser líquidos estos términos son admitidos; por ejemplo, el humor acuoso es producido $(0,2 \mathrm{at} 0,3 \mathrm{ml})$ por el epitelio de los procesos ciliares (9), el líquido cerebroespinal (150 $\mathrm{ml}$ ) es producto fundamentalmente por un filtrado capilar (10), la endolinfa y la perilinfa son los principales líquidos del oído interno (11).

Existen así mismo líquidos como el peritoneal en un volumen de $100 \mathrm{ml}$ aproximadamente que se encuentra en un espacio virtual entre las membranas parietal y visceral de la cavidad peritoneal (12), el líquido pericárdico se estima en una cantidad de 15 a $35 \mathrm{ml}$ de promedio (13) y se estiman producto de filtrados plasmáticos, lo mismo sucede con el líquido pleural (14) con el líquido vaginal, orina y la bilis (15), el semen es producto de la secreción de las glándulas genitales masculinas (16), el sudor y las lágrimas son componentes de la homeostasia hídrica y se expresan en cantidades variables.

El conjunto de líquidos incorporados corresponde a líquidos biológicos necesarios para procesos diversos y tienen funciones de variados efectos en la estructura, al igual que los mencionados en la Tabla 1 , todos dependientes del metabolismo de la sangre.

Por su parte, los términos propuestos, todos sustantivos, tienen identidad semiótica en latín y pueden ser declinados en 1ra: lacrima-ae, saliva-ae, urina-a y en 3ra declinación: bilis, semen, sudor ; por esta razón todos los términos admiten ser adjetivados y formar palabras que clínicamente son aceptables: bilioso, seminal o sudoroso; además sus lexemas acompañados de prefijos o sufijos pueden usarse en denominaciones clínicas o patológicas como hiperbilirrubinemia, bilirragia, seminoma, sudorípara; a su vez los genitivos pleuralis, pericardialis, peritonealis y vaginalis corresponden a la 3ra declinación y son compatibles con pleura visceralis (A07.1.02.002), cavitas pericardiaca (A07.1.02.002) cavitas peritonealis (A10.1.02.001) y con tunica vaginalis testis (A09.3.01.008); estos implican la existencia de espacios virtuales (cavidad pleural, cavidad pericárdica y cavidad peritoneal).

Todos los líquidos biológicos son producto de fenómenos bioquímicos y hemodinámicos, necesarios y básicos para mantener la homeostasia y se expresan permanentemente en condiciones normales en el ser humano en el lenguaje biomédico. Estos líquidos también son fundamentales para mantener la volemia, el equilibrio iónico y/o ácido básico y sus denominaciones son útiles para definir otros estados nosológicos a partir de sus raíces terminológicas.

\section{CONCLUSIÓN}

La definición de líquidos biológicos es clara, todos son productos de un proceso hemodinámico o resultado del metabolismo. La controversia del presente documento es atender la necesidad de mejorar la descripción de TA y no excluir nombres de componentes líquidos que aclaran y completan la descripción de la estructura humana en su totalidad; escoger unos términos y desconsiderar a otros significa desconocer que todos son imprescindibles para la vida. Caso contrario, sería necesario establecer un límite entre lo morfológico y lo fisiológico, definirlo e incorporar estos criterios en TA o considerarlo en sus próximas publicaciones. La selección de unos líquidos sin establecer el criterio previo, motivó este documento que propone la inclusión de este nuevo grupo de términos para completar el listado; por tal razón se debe considerar la creación de un anexo a TA que no altere mayormente los propósitos de respetar la tendencia de disminuir el número de términos; este anexo permitirá admitir todos los elementos, incluidos los líquidos, considerados vitales hasta que eventualmente se proponga la elaboración de una terminología fisiológica.

\section{REFERENCIAS BIBLIOGRAFICAS}

1. Sakai T. Historical evolution of anatomical terminology from ancient to modern. Anat Sci Int. 1 de junio de 2007;82(2):65-81.

2. Algieri RD. Reseña de la evolución histórica de los términos anatómicos. Rev. Arg.Anat.Onl. 2011; 2:106-11.

3. Losardo RJ, Cruz Gutiérrez R, Rodríguez Torres A, Prates JC, Prates NEVB de. Simposios Ibero-latinoamericanos de Terminología Morfológica (SILAT): Primeros Dos Años y Normativas Vigentes. Int $\mathrm{J}$ Morphol. diciembre de 2010;28(4):1323-6.

4. Glare PGW. Oxford Latin Dictionary. Oxford: Oxford University Press; 1968.

5. Thieme G. Terminología Anatómica. International Anatomical terminology. Stuggart. 2001.

6. Thieme G. Terminología Anatomica. International Anatomical. 2da. Ed. Stuggart. 2011.
7. Thieme G. Terminología Histológica International Anatomical terminology. Stuggart. 2006.

8. Rosse C. Terminologia Anatomica: Considered from the perspective of nextgeneration knowledge sources. Clin Anat. 2001;14(2):120-33.

9. Feneis, H. \& Dauber, W. Pocket Atlas of Human Anatomy. Based on the International Nomenclature. 4th ed. Stuttgart: Thieme; 2000 .

10. Sakka L, Coll G, Chazal J. Anatomy and physiology of cerebrospinal fluid. Eur Ann Otorhinolaryngol Head Neck Dis. diciembre de 2011;128(6):309-16.

11. Suárez C, Gil-Carcedo LM, Algarra JM, autores. Tratado de otorrinolaringología y Cirugía de Cabeza y Cuello. 2da. ed. Tomo II. Madrid: Panamericana; 2007.

12. Pannu HK, Oliphant M. The subperitoneal space and peritoneal cavity: basic concepts. Abdom Imaging. octubre de 2015;40(7):2710-22

13. Vogiatzidis K, Zarogiannis SG, Aidonidis I, Solenov EI, Molyvdas P-A, Gourgoulianis KI, et al. Physiology of pericardial fluid production and drainage. Front Physiol [Internet].2015 [citado 10 de noviembre de 2020];6. Disponible en: http://www. frontiersin.org/Membrane Physiology and Membrane Biophysics/10.3389/ fphys.2015.00062/abstract.

14. Charalampidis C, Youroukou A, Lazaridis G, Baka S, Karavasilis V, Kioumis I, et al. Physiology of the pleural space. J Thorac Dis. 2015; 7:5.

15. Hermine C. L.M.D. Radiology of Liver Circulation Series in Radiology. Kindle. Vol. 11. 1985. 1-3 p.

16. Strasinger, Di Lorenso. Análisis de orina y de líquidos corporales. 5a ed. China: Panamericana; 2010. 